\title{
Surgical outcome for rhinogenic contact point headache in Rizgary Teaching Hospital in Erbil, Iraq
}

\begin{abstract}
Background and objective: The definition of an intranasal contact point is when two regions within the nasal cavity are opposing each other and resist separation following the application of a topical decongestant. A contact point should be identified by endoscopy, but some authors have used the appearance on computed tomography for the selection criteria. This study aimed to assess the role of some anatomical variations of the nose in rhinogenic contact point headache and to determine the role of surgery in the management of such headache.

Methods: This prospective (case-series) study included 30 patients from Rizgary Teaching Hospital in Erbil city, Iraq during the period of March 2015 to March 2017. All patients involved in this study were having symptoms for at least more than one year and not responding to medications.

Results: The study participants were 18 females and 12 males; their ages range between 18 and 43 years with a mean age of 26.90 years. Regarding surgical outcome, all patients underwent appropriate surgery and followed up for at least six months postoperatively. Twenty seven $(90 \%)$ patients had a complete cure, three $(10 \%)$ patients with ties or remaining the same and none $(0 \%)$ with positive ranks.

Conclusion: The removal of contact points in patients with Rhinogenic Contact Point Headache is very effective for relieving the pain in carefully selected patients.

Keywords: Rhinogenic headache; Contact points; Deviated nasal septum.
\end{abstract}

\section{Introduction}

It is not so uncommon in the setting of an office to encounter a patient complaining of severe recurrent pain localized to the glabella, supraorbital region, or eyes without any clinical or radiological signs of rhinosinusitis. These unfortunate patients usually pay multiple visits to otorhinolaryngology, neurology, and ophthalmology clinics where their examinations reveal normal findings. Not too infrequently, they may be missdiagnosed and treated for rhinosinusitis, migraine or vascular-type headaches. ${ }^{1}$ Patients with facial pain are frequently referred to otorhinolaryngologists. Most patients arrive in the clinic with an initial diagnosis of sinusitis, although in reality few of these patients have sinogenic pain.
Reaching the correct diagnosis in patients with facial pain is challenging because many patients come with fixed ideas about the cause of their pain. ${ }^{2}$ Stimulation of the lateral wall of the nose through contact between middle turbinate and nasal septum in cases of severely deviated nasal septum, enlarged pneumatized middle turbinate (concha bullosa), or medially displaced middle turbinate by enlarged ethmoidal bulla produce contact edema between mucosal surfaces and release of pain mediators resulting in pain radiating along nerve fibers. ${ }^{3}$ In 2004, mucosal contact headache was added as a secondary headache disorder in the International Classification of Headache Disorders. The guide described mucosal contact headache as variations in mucosal

${ }^{1}$ Teaching Center for Otolaryngology, Head and Neck Surgery, Erbil, I raq.

* Correspondence: botanent3@yahoo.com 
congestion mediated by gravitational changes. The criteria in the guide require the abolition of a headache within 5 minutes following the application of topical anesthesia to the contact point area and significant improvement of a headache in less than seven days after removal of the mucosal contact points. ${ }^{4}$ Various surgical modalities are done for the management of a headache, e.g., septoplasty and partial middle turbinectomy. ${ }^{5}$ Due to the debates regarding this problem, this study aimed to evaluate the effect of various anatomical structures in the pathogenesis of a headache and the outcomes of its surgical management.

\section{Methods}

This prospective study has been conducted at Otolaryngology Department of the Rizgary Teaching Hospital in Erbil city, Iraq from $7^{\text {th }}$ March 2015 to $12^{\text {th }}$ March 2017. This study included 30 patients (12 males and 18 female); their age ranged from 18 to 43 years. These patients presented with a complaint of nasal obstruction and headache of rhinogenic origin of more than one year duration. All patients had a long-standing history of headaches, and none had ever experienced satisfactory relief from any previous attempts of treatment. All patients underwent a preoperative evaluation including detailed medical history, a complete ENT examination, diagnostic nasal endoscopy, and radiological imaging to assess anatomical variations. Patients were asked about the use of analgesics or other drugs and their adequacy for alleviating their complaints. After application of topical decongestant as xylometazoline, $0.1 \%$ to whole the nose and a small piece of cotton soaked in lidocaine $2 \%$ applied to the contact points by the aid of an endoscope. Those patients who had clear contact points in their nasal septa by endoscopy and whose headaches had responded to this application were included as a positive anesthetic test. All patients were sent for neurological, ophthalmological, and dentistry consultation. The preoperative headache intensity was recorded based on a 10-point visual analog scale (VAS) in which 0 symbolizes the absence of a headache, and 10 represents the most intense pain of all (Figure 1). ${ }^{6}$ Then all the patients underwent necessary intranasal operations under general anesthesia, and all of the patients were followed up for six months postoperatively. The inclusion criteria include duration of a headache for more than one year, not responding to several trials of painkiller, presence of contact between the part of the lateral wall of the nose and septum on clinical and endoscopic examination and in CT images, positive local anesthesia test, normal sinus CT scan, and normal Neurological examination and consultation. The exclusion criteria included a short duration of a headache of less than one year, a patient is cured with analgesia, contact between the lateral wall of the nose and the nasal septum is not confirmed by either clinical, endoscopic or CT imaging, negative local anesthesia test, sinus infection on CT Scan and diagnostic as a neurological case.

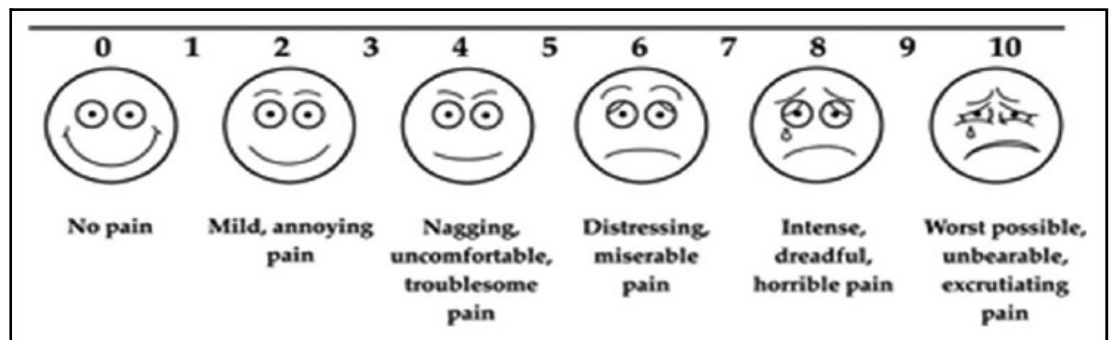

Figure 1: Headache and pain scale used in the study. 
Postoperative care and follow up

Patients were followed up on a monthly basis for six months. Patients were encouraged to report on the current status of their headache symptoms and any interval change either at the time of hospital visit follow up or by phone calls. In every visit of the patient, nasal endoscopy was performed.

\section{Ethical considerations}

Informed and written consent was taken from each patient after explaining the purpose of the study by the researcher.

Data entry and statistical analysis:

Data were analyzed using the statistical package for the social sciences. Wilcoxon signed ranks test was used to compare ranks of pain score pre and postoperatively. A $P$ value of $\leq 0.05$ was considered statistically significant.

Criteria for Cure, Improvement, and Failure:

Postoperative $6^{\text {th }}$-month patients were assessed by VAS. Complete cure was considered if the patient was completely free of his preoperative contact point headache. Improvement was considered if the attacks became less frequent and less severe by $70 \%$ than an original headache. Otherwise, failure was considered.

\section{Results}

This study included 30 patients complaining of contact headache of more than one-year duration and resistant to medical treatment. The mean \pm SD age was $26.90 \pm 7.78$ (range 18 to 43 years). Eighteen of them were female $(60 \%)$, and 12 were male (40\%). All 30 patients had clear contact point by either anterior rhinoscopy and flexible or rigid endoscopy; $18(60 \%)$ of them located on the left side while $12(40 \%)$ of them located on the right side. Out of 30 patients, only 23 patients $(76.7 \%)$ had CT scan assessment preoperatively to detect variable anatomical findings and seven patients $(23.3 \%)$ without doing a preoperative CT assessment because contact points diagnosed clinically, were not needing imaging for an economic state. Therefore, the findings were missing (Table 1).

Table 1: Findings of anatomical variation of CT scan of PNS.

\begin{tabular}{lccc}
\hline Variables & Categories & Number & Percent \\
\hline Deviated nasal septum & Right & 12 & 40 \\
& Left & 11 & 36.7 \\
Hypertrophied middle turbinate & None & 18 & 60 \\
& Left & 5 & 16.7 \\
Concha bullosa & None & 17 & 56.7 \\
& Right & 2 & 6.7 \\
Double middle turbinate & Left & 4 & 13.3 \\
Paradoxical middle turbinate & None & 23 & 76.7 \\
Hypertrophied inferior turbinate & None & 23 & 76.7 \\
& None & 4 & 13.3 \\
& Right & 7 & 23.3 \\
Cases with CT findings & Left & 6 & 20 \\
Without CT findings & Bilateral & 6 & 20 \\
Total & & 23 & 76.7 \\
\hline
\end{tabular}


All 30 patients $(100 \%)$ had undergone successful surgical procedures and without complications; 18 patients $(60 \%)$ with septoplasty and submucous diathermy, six patients $(20 \%)$ with septoplasty only, four patients $(13.4 \%)$ with septoplasty and conchoplasty and submucous diathermy, and two patients $(6.6 \%)$ with septoplasty and conchoplasty. All 30 patients (100\%) were evaluated postoperatively by pain evaluation scale for at least six months (Figure 2). Out of 30 patients, 27 of patients $(90 \%)$ had an improvement of a headache and only three patients (10\%) remained with the pain postoperatively (Table 2).

Table 2: Comparison between pre and post-operative pain scores. *

\begin{tabular}{lcccccc}
\hline Pain score & N & Mean & S.D & Wilcoxon test & $P$ value \\
\hline Pre-op score & 30 & 5.40 & 1.49 & Negative Ranks & 27 & \\
& & & & Positive Ranks & 0 & $<0.001$ \\
& & & & Ties & 3 & \\
Post-op score & 30 & 0.93 & 1.79 & Total & 30 & \\
\hline
\end{tabular}

* Wilcoxon Signed Ranks Test was used for comparison.

$\mathrm{N}$ : Number

S.D: Standard Deviation

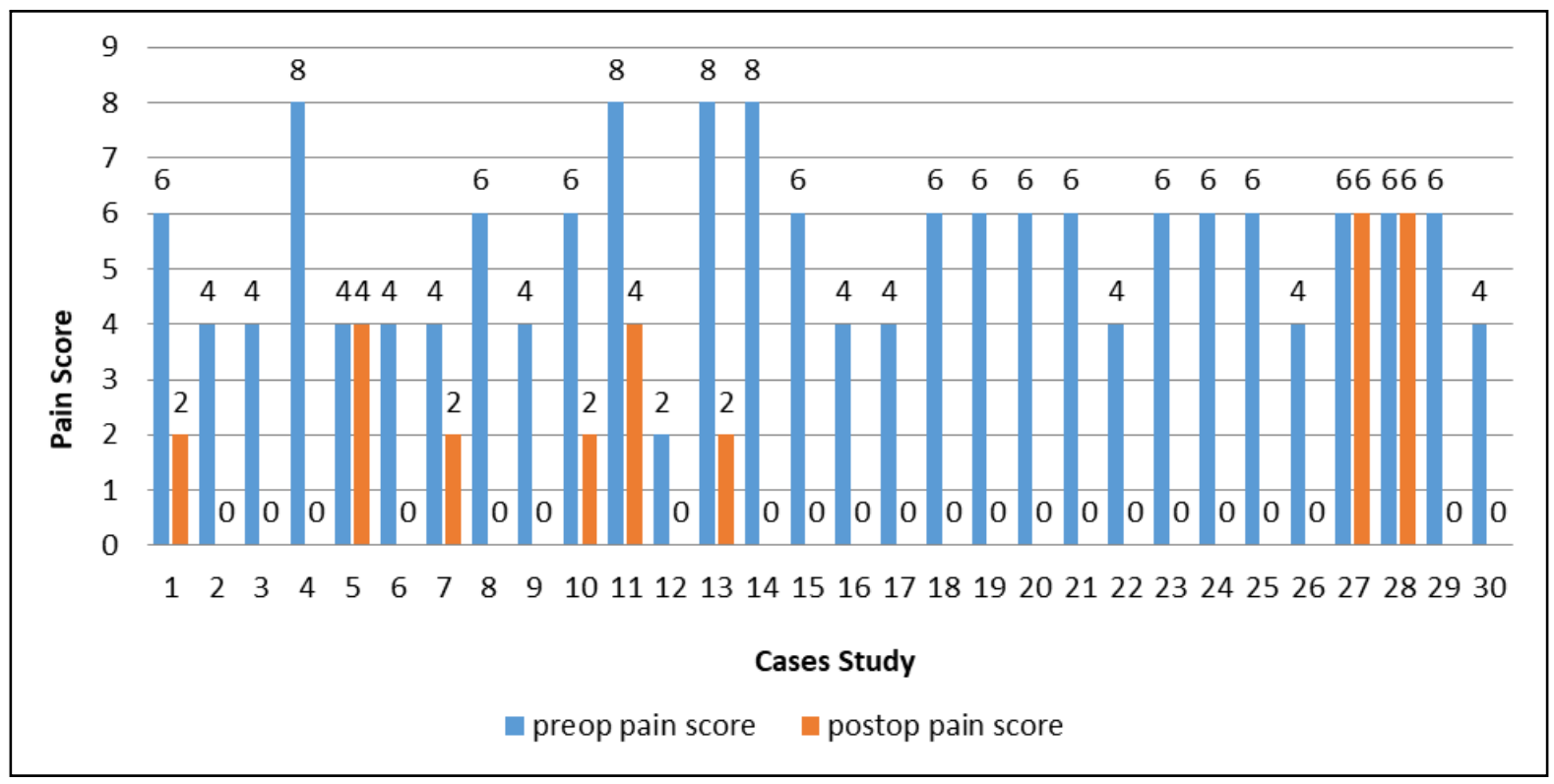

Figure 2: Comparison between preoperative and postoperative pain scale. 


\section{Discussion}

Over the years, many theories have been proposed to explain the exact pathophysiology of primary headaches with a possible nasal origin until the advent of nasal endoscopy and computed tomography scanning (CT) has greatly challenged this problem. Besides their resistance to the ordinary medical anti-headache measures, rhinogenic headache patients usually show underlying endoscopic and/or radiological sinonasal changes in the form of anatomic variations and/or mucosal disease. ${ }^{7}$ The exact mechanism of this type of headache is unclear. The prominent theory is the production of different neuropeptides in areas of contact mucosal points. The most famous neurotransmitter is the $P$ substance, and the most common place of production is septum and middle concha. ${ }^{8}$ In the current study, the examination of patients during the headache attack showed that mucosal contact was present between the septum and a part of the lateral wall of the nose in all the cases. This matches with the study of Abdel Tawab et al., ${ }^{9}$ who reported that septal-turbinate contact implies that the pathologic process in rhinogenic headache is in the nasal rather than the sinus cavity. However, it is in contrast to the study by Behin et al. ${ }^{10}$ were enrolled patients with the only contact between the septum, superior turbinate and/or ethmoid and the patients who demonstrated contact between the septum and middle turbinate on CT were excluded. By clinical and endoscopic examination, we found that various anatomical variations had been implicated as possible causes of rhinogenic headache in the absence of sinusitis. In this issue, $100 \%$ of patients had deviated nasal septum. This matches with the study of Ramadan et al. ${ }^{11}$ and Hammad et al., ${ }^{12}$ who reported patients with headache secondary to septal deviation. Also, hypertrophied inferior turbinate variations $(73.4 \%)$ were the next commonest causes. This finding comes in agreement with the study of Sadeghi et al. ${ }^{13}$ that showed improvement of headaches after correction. In the current study, all 30 $(100 \%)$ patients of contact point selected by clinical and endoscopic examination, while only 23 (76.7) patients had preoperative CT scan for evaluation of other anatomical variations, and seven $(23.3 \%)$ patients were missing because of resources and economic state. Needless to say that all the aforementioned stages (CT scan, nasal endoscopy, lidocaine test) were necessary for validating the rhinogenic source of the headache because of the fact that nasal cycle can mimic any form of contact points which are not permanent. ${ }^{14}$ In the current study, only patients were enrolled with a positive local anesthetic test, and negatives were excluded. Many cases with contact points detected by clinical examination and CT findings but with a negative local anesthetic test. Lidocaine test plays an essential role in the final identification of the rhinogenic headache. ${ }^{15}$ This differs with the study of Mokbel et $\mathrm{al}^{16}$ where patients enrolled with both lidocaine positive and negative results, where enrolled patients with negative lidocaine test who get benefit from the surgery. Pain is subjective, and a known tissue injury or nerve lesion is not necessary for the pain to be experienced Although the most reliable indicator for the presence and severity of pain is the patient's self-report, the decision to administer pain-relieving therapy should not be based exclusively on the patient's ability to communicate. ${ }^{17}$ On the other hand, a negative local anesthetic test does not rule out the diagnosis of a contact point headache in all cases. Patients with a typical pain are presumably more sensitive to minor mucosal injury reflecting the importance of localized endoscopic resection with less tissue trauma. ${ }^{18}$ In the current study, all the enrolled patients were having a normal neurological evaluation preoperatively by consulting neurological physician, and any positive neurological findings were excluded from the study and 
all the patients were followed up at least six months postoperatively. This is in disagreement with the study of Behin et al., ${ }^{19}$ who enrolled patients with a refractory migraine and the longer time of patients follow up. In the current study, 30 (100\%) patients had undergone the appropriate intra-nasal operation and after follow up for at least six months were showed that $90 \%$ of patients were cured and $10 \%$ were unchanged. The findings come in agreement regarding the postoperative success rate with both studies by Luessen et al. ${ }^{20}$ and Sindwani et al. ${ }^{21}$ while disagreement with the study of Abu-Samra et al., ${ }^{22}$ that showed $19 \%$ had complete cure and $81 \%$ still had residual symptoms. In the current study, the failed cases showed a very long history of headache and longer duration of every single attack of headache. As the duration of headache complaint or the duration of every attack increases, it will be unlikely for the headache to be cured as soon after surgery. Also, any changes affecting the social, psychiatric, and physical conditions of the patient after surgery can endanger the results of surgery.

\section{Conclusion}

Patients with facial pain and headache should be investigated carefully to show the exact cause. Lidocaine can be used as a test to aid in the diagnosis of this complaint; when it is positive, can be considered as an indicator of the success of the surgery. The current study showed that the surgical removal of the contact points alleviates a headache. Despite the benefit of the surgery for rhinogenic contact point headache and follow up for short time duration while in some recent long-term studies for 10-20 years, showed that long-term follow-ups is recommended to identify the pattern of the recurrence after treatment.

\section{Competing interests}

The authors declare that they have no competing interests.

\section{References}

1. Devrim B, Zekeriya A, Nurettin A, Ahmet U, Osman B, Refik C. Surgical Outcomes for Rhinogenic Contact Point Headaches. Med Princ Pract 2011; 20:29-33.

2. Woolford TJ, Nicholas SJ. The diagnosis and management of facial pain. Michael Gleeson. Scott-Brown's Otorhinolaryngology, Head and Neck Surgery. $7^{\text {th }}$ ed. Great Britain: Hodder Arnold; 2008. P. 1718.

3. Stammberger $H$, Wolf $G$. Headache and sinus diseases. The endoscopic approach. Ann Otol Rhinol Laryngol Suppl 1988; 134:3-23.

4. Headache Classification Committee of the International Headache Society: The international classification of headache disorders. Cephalalgia 2004; 24(suppl 1):1-160.

5. Low WK, Willatt DJ. Headaches associated with nasal obstruction due to deviated nasal septum. Headache 1995: 35(7):04-6.

6. Christine Miserandino: How do you describe your pain? (Accessed April 30, 2017, at https://butyoudontlooksick.com/articles/ pain_description/).

7. Houseny SE, Yaser KL, Ayman A, Mohamed S. Surgical Outcomes for Rhinogenic Contact Point Headaches. Menoufiya Medical Journal 2011; 24(2):71-82.

8. Abu-Bakra $M$, Jones $N$. Does stimulation of nasal mucosa cause referred pain to the face. Clin Otolaryngol Allied Sci 2001; 26:430-2.

9. Abdel Tawab HM, Gharib FM, Raafat SA, Mohamed $\mathrm{MH}$. Surgical Outcomes for Rhinogenic Contact Point Headaches. PAN Arab J Rhinol 2014; 4(1):2-8.

10. Behin F, Behin B, Bigah E, Lipton SRB. Surgical treatment of patients with refractory migrain headaches and intranasal contact points. Cephalgia 2004; 14(5):121-7.

11. Ramadan HH. Nonsurgical versus endoscopic sinonasal surgery for rhinogenic headache. Am J Rhinol 1999; 13:455-7.

12. Hammad MS, Gomaa MA. Role of some anatomoical nasal abnormalities in rhinogenic headache. Egyptian journal of Ear, Nose, Throat and Allied Sciences 2012; 13:31-5.

13. Sadeghi M, Saedi B, Ghaderi Y. Endoscopic management of contact point headache in patient's resistant to medical treatment. Indian J Otolaryngol Head Neck Surg 2013; 65(suppl 2):415-20.

14. Mehle M, Kremer P. Sinus CT scan findings in "sinus headache" migraineurs. Headache 2008; 48:67-71.

15. Mohebbi A, Memari F, Mohebbi S. Endonasal endoscopic management of contact point headache and diagnostic criteria. Headache 2009; 50:242-8.

16. Mokbel KM, Abd Elfattah AM, El-Sharawy K. Nasal mucosal contact points with facial pain 
and/or headache: lidocaine can predict the result of localized endoscopic resection. Eur Arch Otorhinolaryngol 2010; 267:1569-72.

17. Bottros MM, Rao L, Schwedt TJ, Swarm RA. Pain management. Paul W Flint. Cumming otolaryngology, Head and Neck Surgery. $6^{\text {th }}$ ed. Canada: Saunders Elsevier; 2015. P. 240.

18. Chow JM. Rhinologic headaches. Otolaryngol Head Neck Surg 1994; 111:211-8.

19. Behin F, Behin B, Behin D, Baredes S. Surgical management of contact point headaches. Headache 2005; 45:204-10.

20. Welge-Luessen A, Hauser R, Schmid N, Kappos L, Probst R. Endonasal surgery for contact point headaches: a 10-year longitudinal study. Laryngoscope 2003; 113(12):2151-6.

21. Sindwani R, Wright ED. Role of endoscopic septoplasty in the treatment of atypical facial pain. J Otolaryngol 2003; 32:77-80.

22. Abu-Samra M, Gawad OA, Agha M. The outcomes for nasal contact point surgeries in patients with unsatisfactory response to chronic daily headache medications. Eur Arch. Otorhinolaryngol. 2011; 268:1299-304. 